\title{
A influência da incontinência urinária na qualidade de vida de mulheres jovens: uma
}

\section{revisão de literatura}

\author{
The influence of urinary incontinence on the quality of life of Young women: literature review \\ La influencia de la incontinencia urinaria en la calidad de vida de las mujeres jovenes: revisión de \\ la literatura
}

Recebido: 24/09/2021 | Revisado: 30/09/2021 |Aceito: 01/10/2021 | Publicado: 03/10/2021

\author{
Bruna Ribeiro de Souza \\ ORCID: https://orcid.org/0000-0002-4645-7773 \\ Centro Universitário da Amazônia, Brasil \\ E-mail: ribeirobfisio@gmail.com \\ Isabely dos Santos Dantas \\ ORCID: https://orcid.org/0000-0003-0762-7984 \\ Centro Universitário da Amazônia, Brasil \\ E-mail: isabelydantas99@gmail.com \\ Janglea Sousa de Souza \\ ORCID: https://orcid.org/0000-0003-4167-4274 \\ Centro Universitário da Amazônia, Brasil \\ E-mail: janglea.sousa97@hotmail.com \\ Indiara de Alencar \\ ORCID: https://orcid.org/0000-0003-3204-3278 \\ Universidade do Estado do Pará, Brasil \\ E-mail: indiara.alencarstm@gmail.com
}

\begin{abstract}
Resumo
A incontinência urinária (IU) está entre uma das situações que diminuem a qualidade de vida das mulheres, devido ao comprometimento em várias áreas da vida, o que deixa a mulher vulnerável a vários problemas por conta das restrições que as limitam. A incontinência urinária não é uma patologia específica de mulheres idosas, visto que o número de mulheres jovens com essa disfunção tem se tornado relevante afetando as mulheres em diversas fases do ciclo vital. A IU está entre uma das situações que diminuem a qualidade de vida das mulheres, devido ao comprometimento em várias áreas da vida, como físico, social e principalmente psicológico, o que leva a limitação de atividades sociais e familiares. Devido a falta de informação, muitas mulheres acham que não existe tratamento e imaginam que é normal esse escape de urina. Como metodologia do estudo buscou-se reunir artigos que apresentaram importância no campo científico sobre o tema proposto. Apesar de serem poucos o número de estudos encontrados, podemos concluir que a incontinência urinária afeta de forma negativa a qualidade de vida das mulheres, principalmente no que diz respeito ao âmbito psicológico, incapacitando-as de realizar de forma adequada suas atividades de vida diária.
\end{abstract}

Palavras-chave: Incontinência urinária; Saúde da mulher; Qualidade de vida.

\begin{abstract}
Urinary incontinence (UI) is among the situations that reduce the quality of life of women, due to impairment in various areas of life, which leaves women vulnerable to various problems due to the restrictions that limit them. Urinary incontinence is not a specific pathology of elderly women, as the number of young women with this disorder has become relevant, affecting women at different stages of the life cycle. UI is among the situations that reduce the quality of life of women, due to impairment in various areas of life, such as physical, social and especially psychological, which leads to limitation of social and family activities. Due to lack of information, many women think that there is no treatment and imagine that this leakage of urine is normal. As a study methodology, we sought to gather articles that were important in the scientific field on the proposed topic. Despite the few studies found, we can conclude that urinary incontinence negatively affects the quality of life of women, especially with regard to the psychological sphere, making them unable to adequately perform their daily activities.
\end{abstract}

Keywords: Urinary incontinence; Women's health; Quality of life.

\section{Resumen}

La incontinencia urinaria (IU) es una de las situaciones que merma la calidad de vida de la mujer, por deterioro en diversos ámbitos de la vida, lo que deja a la mujer vulnerable a diversos problemas por las restricciones que la limitan. La incontinencia urinaria no es una patología específica de las mujeres mayores, ya que el número de mujeres jóvenes 
con este trastorno ha cobrado relevancia, afectando a mujeres en diferentes etapas del ciclo de vida. La IU se encuentra entre las situaciones que merman la calidad de vida de la mujer, por deterioro en diversos ámbitos de la vida, como el físico, social y especialmente psicológico, lo que lleva a la limitación de las actividades sociales y familiares. Por falta de información, muchas mujeres piensan que no existe tratamiento e imaginan que esta fuga de orina es normal. Como metodología de estudio, se buscó recopilar artículos de relevancia en el campo científico sobre el tema propuesto. A pesar de los escasos estudios encontrados, podemos concluir que la incontinencia urinaria afecta negativamente a la calidad de vida de las mujeres, especialmente en lo que respecta al ámbito psicológico, haciéndolas incapaces de realizar adecuadamente sus actividades diárias.

Palabras clave: Incontinencia urinaria; Salud de la mujer; Calidad de vida.

\section{Introdução}

Atualmente a Sociedade internacional de continência define a incontinência urinária (IU) como toda e qualquer perda de urina. A IU é classificada em 3 tipos, sendo Incontinência urinária de esforço (IUE), definida como toda e qualquer perda de urina associada a algum esforço, como tosse, espirros, carregar objetos, atividade física entre outros, Incontinência Urinária de urgência (IUU) é quando a perda de urina está adjunta a necessidade imediata de urinar e a Incontinência urinaria mista é caracterizada pela associação da perda de urina à urgência e ao esforço (Abrams., 2013).

De acordo com Liao et al. (2009) em território nacional a prevalência ainda é inexplorada, visto que as pesquisas realizadas foram concentradas apenas em grandes centros, como São Paulo, assim não podendo ser considerado um dado fidedigno para o todo território brasileiro. Ainda assim, nota-se que a prevalência de IU aumenta significativamente de acordo com a idade, sendo que 26,5\% de IU são em mulheres acima dos 20 anos, de 49,6\% em mulheres com idade de 35 a 64 anos e $41 \%$ em mulheres acima de 65 anos (Leroy, et al., 2012).

Quanto aos fatores etiológicos mulheres obesas são cerca de 4,2 vezes mais acometidas pela IU, em comparação a mulheres que tem massa corporal normal, e a idade pode estar relacionada a diminuição das fibras de colágeno e modificação do tecido muscular por gordura, além das alterações hormonais como diminuição do estrogênio que é responsável pela coaptação da uretra. O período gestacional e paridade acarretam no aumento da pressão mecânica no assoalho pélvico, podendo levar a incontinência urinária. Outros fatores que levam a IU são, tabagismo, diabetes, sedentarismo, alcoolismo, hereditariedade, constrição intestinal, fármacos, consumo de cafeína e afecções neurológicas (Salama, et al., 2015).

Nos últimos anos, tem-se aumentado as pesquisas quanto a qualidade de vida em diferentes contextos e áreas de atenção à saúde, a qualidade de vida está relacionada ao grau de satisfação no meio social, vida amorosa, vida familiar e valores existenciais. A OMS afirma que, a qualidade de vida reflete a posição da mulher na vida, seja no contexto de cultura, padrões, objetivos e ou preocupações (Rett et al, 2007).

Devido ao comprometimento em várias áreas da vida, a mulher fica vulnerável a vários problemas por conta das restrições que as limitam (Pedro, et al., 2011). A IU tende a afetar a condição física das mulheres, o que leva a limitação de atividades sociais e familiar, e pela falta de informação, muitas mulheres acham que não existe tratamento e imaginam que é normal esse escape de urina, há uma relutância também na busca do tratamento e estas mulheres acabam se isolando para evitar certos constrangimentos, o que acaba influenciando para uma baixa qualidade de vida (Fernandes, et al., 2015).

O tratamento conservador da IU abrange o acompanhamento clínico, por meio da administração de fármacos que causam contração ou relaxamento vesico-uretral, além do acompanhamento fisioterapêutico, considerado tratamento de primeira linha para as incontinências leves e moderadas (Marques et al, 2017). Lembrando que os tratamentos ofertados pelo SUS para a IU são os cirúrgicos e os medicamentosos, já os fisioterápicos, muitas vezes são por iniciativa das pacientes em algum órgão privado ou por algum serviço de reabilitação de universidades (Delarmelindo et al, 2012).

Segundo a International Continence Society (ICS), o Treinamento dos Músculos do Assoalho Pélvico (TMAP) devem ser a primeira escolha de terapia para mulheres com Incontinência Urinária (Berghmans et al., 2015). De modo geral, o tratamento fisioterapêutico visa a prevenção e o tratamento curativo da IU, através da reeducação da função miccional, 
informando quanto ao uso adequado da musculatura do assoalho pélvico, bem como ensinando técnicas de exercícios para o fortalecimento dessa musculatura, uma vez que na maioria dos casos de incontinência urinária, está presente a redução de força dessa região. E além dos exercícios através da cinesioterapia, podem ser citados algumas outras formas de tratamento como, biofeedback, terapia comportamental e eletroestimulação neuromuscular (Oliveira \& Gracia, 2011).

\section{Metodologia}

O método científico se inicia na observação organizada de fato, da realização de experiências, das deduções lógicas e da comprovação científica dos resultados adquiridos. Por tanto, é o caminho que se deve adotar para levar a formulação de uma teoria científica (Pereira et al, 2018).

A construção do presente estudo fundamentou-se em uma revisão bibliográfica de caráter descritivo qualitativo. Para conduzir a elaboração dessa revisão determinamos como a questão problema: "Mulheres jovens que apresentam quadro de incontinência urinária, possuem a qualidade de vida afetada?"

Para elaboração do presente estudo buscou-se reunir artigos que se apresenta importância no campo científico sobre o tema proposto, foi realizado por meio de cinco itens, que são: definição de uma pergunta norteadora, seleção as bases de dados, filtragem os artigos, avaliação os estudos, construção os resultados e discussões. A pesquisa foi executada nas seguintes bases eletrônicas: Google Acadêmico, Physiotherapy Evidence Database (PEDro) e Scientific Electronic Library Online (SciELO), utilizando os descritores: Incontinência urinária, saúde da mulher, qualidade de vida.

A análise foi feita a partir da leitura criteriosa dos artigos selecionados. Com o total de 9 estudos, onde foi verificado a influência da incontinência urinária na qualidade de vida de mulheres jovens.

Os critérios de inclusão foram trabalhos que tenham sido publicados entre os anos 2014 a 2021, com estudos randomizados que abordassem a temática sobre a incontinência urinária e a influência da mesma na qualidade de vida de mulheres jovens, publicações na íntegra e de acesso livre, e estudos publicados na língua portuguesa e inglesa.

Como critérios de exclusão foram artigos não disponibilizados na íntegra, trabalhos duplicados, publicações anteriores ao ano de 2014, artigos que não tem coerência com o tema proposto, trabalhos não concluídos e de revisão bibliográfica e artigos pagos. 
Figura1. Fluxograma da metodologia de pesquisa.

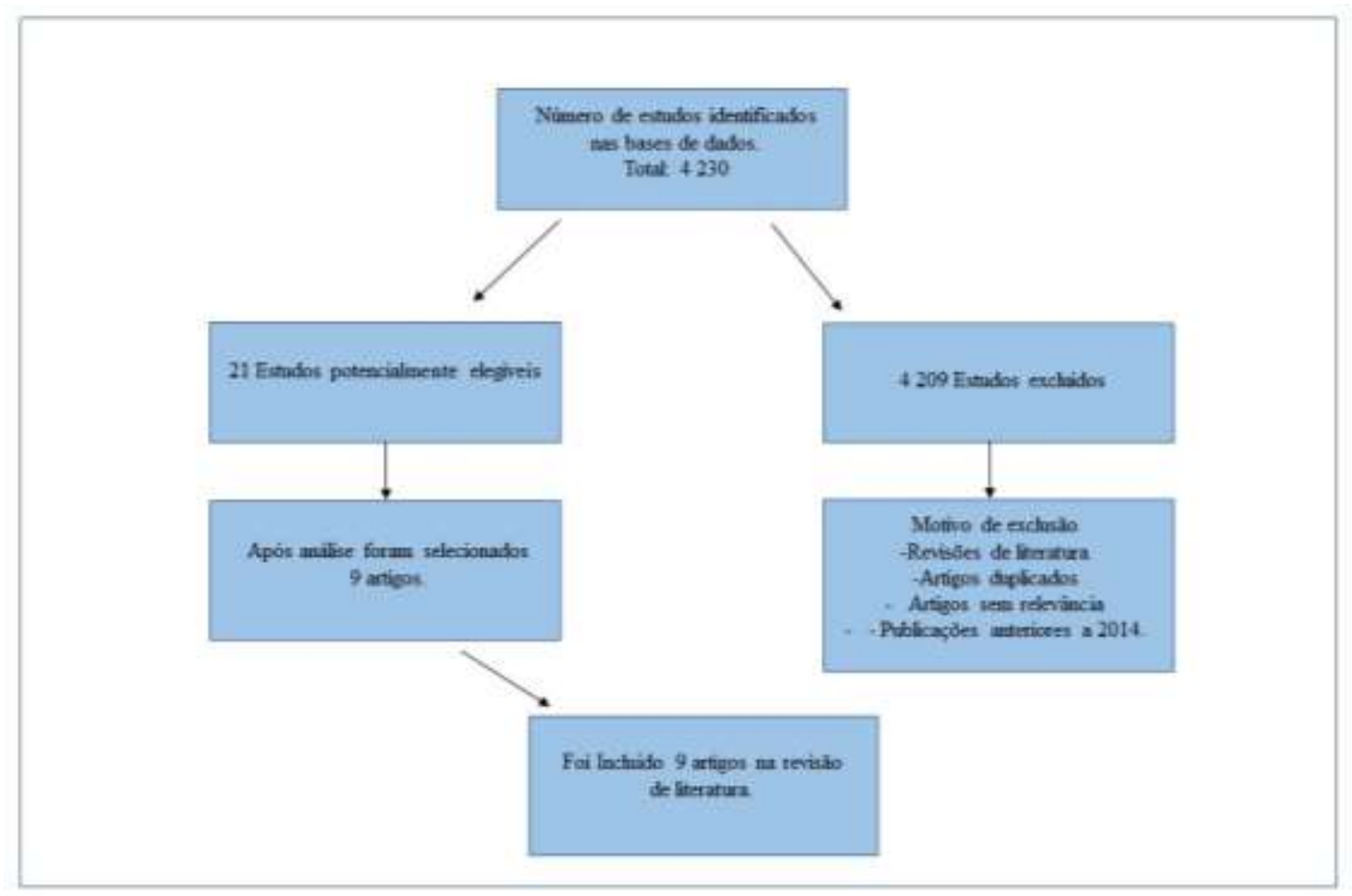

Fonte: Souza, Souza, Dantas e Alencar (2021).

\section{Resultados e Discussão}

No Quadro 1 mostra-se 9 estudos, voltados para análise da qualidade de vida de mulheres adultas jovens com incontinência urinária.

Quadro 1 - Resultados dos estudos publicados nos anos de 2014 à 2020.

\begin{tabular}{|c|c|c|c|c|c|}
\hline TÍTULO & AUTOR & $\begin{array}{c}\text { AMOSTRA/I } \\
\text { DADE }\end{array}$ & MÉTODO & OBJETIVO & RESULTADOS \\
\hline $\begin{array}{c}\text { Frequência da } \\
\text { incontinência } \\
\text { urinária em } \\
\text { mulheres na } \\
\text { idade } \\
\text { produtiva. }\end{array}$ & $\begin{array}{l}\text { DANTAS, et al., } \\
2020 .\end{array}$ & $\begin{array}{l}300 \text { mulheres } \\
\text { da cidade de } \\
\text { Pilões/RN. } \\
\text { De } 20 \text { A } 59 \\
\text { anos. }\end{array}$ & $\begin{array}{l}\text { Questionário } \\
\text { auto aplicado. }\end{array}$ & $\begin{array}{l}\text { Conhecer a frequência } \\
\text { da incontinência } \\
\text { urinária em mulheres } \\
\text { em idade produtiva e o } \\
\text { conhecimento das } \\
\text { mesmas acerca da IU. }\end{array}$ & $\begin{array}{c}\text { A frequência de incontinência } \\
\text { urinária feminina encontrada } \\
\text { foi de } 8 \% \text { e a idade média foi } \\
\text { de } 38,8 \text { anos. Evidenciando a } \\
\text { frequência da incontinência } \\
\text { urinária em mulheres com } \\
\text { idade produtiva na cidade de } \\
\text { Pilões/RN }\end{array}$ \\
\hline $\begin{array}{l}\text { Prevalência e } \\
\text { Fatores de risco } \\
\text { associados a } \\
\text { incontinência } \\
\text { urinária em } \\
\text { acadêmicas do } \\
\text { curso de } \\
\text { fisioterapia } \\
\end{array}$ & $\begin{array}{c}\text { FERNANDES \& } \\
\text { OGNIBENI., } \\
2020\end{array}$ & $\begin{array}{l}79 \text { mulheres. } \\
\text { Com idade } \\
\text { igual ou } \\
\text { superior a } 18 \\
\text { anos. }\end{array}$ & $\begin{array}{c}\text { ICIQ-SF - } \\
\text { International } \\
\text { Consultation } \\
\quad \text { on } \\
\text { Incontinence } \\
\text { Questionnaire } \\
\text { - Short Form. }\end{array}$ & $\begin{array}{l}\text { Avaliar a prevalência e } \\
\text { os fatores de risco } \\
\text { associados à } \\
\text { incontinência urinária } \\
\text { em acadêmicas do } \\
\text { Curso de Fisioterapia. }\end{array}$ & $\begin{array}{l}\text { Apenas uma parte das } \\
\text { acadêmicas apresentou } \\
\text { incontinência urinária e entre } \\
\text { os fatores de risco, somente a } \\
\text { infecção urinária esteve } \\
\text { associada com a patologia. }\end{array}$ \\
\hline $\begin{array}{l}\text { Comparação da } \\
\text { qualidade de } \\
\text { vida nos } \\
\text { diferentes tipos } \\
\text { de } \\
\text { incontinência } \\
\text { urinária } \\
\text { feminina. }\end{array}$ & $\begin{array}{c}\text { DEDICAÇÃO, et } \\
\text { al., } 2018 .\end{array}$ & $\begin{array}{c}77 \\
\text { voluntárias. } \\
\text { De } 34 \text { a } 85 \\
\text { anos. }\end{array}$ & $\begin{array}{l}\text { King's Health } \\
\text { Questionnaire. }\end{array}$ & $\begin{array}{l}\text { Comparar o impacto de } \\
\text { cada tipo de } \\
\text { incontinência urinária } \\
\text { sobre a qualidade de } \\
\text { vida de mulheres. }\end{array}$ & $\begin{array}{l}\text { O estudo indicou que pacientes } \\
\text { com incontinência urinária } \\
\text { mista apresentaram um maior } \\
\text { impacto negativo sobre a } \\
\text { qualidade de vida. }\end{array}$ \\
\hline
\end{tabular}




\begin{tabular}{|c|c|c|c|c|c|}
\hline $\begin{array}{c}\text { Avaliação da } \\
\text { incontinência } \\
\text { urinária e } \\
\text { qualidade de } \\
\text { vida em } \\
\text { casados }\end{array}$ & $\begin{array}{l}\text { OZDEMIR, et al., } \\
2018 .\end{array}$ & $\begin{array}{c}\text { Em } 1161 \\
\text { mulheres } \\
\text { casadas. De } \\
20 \text { a } 49 \text { anos. }\end{array}$ & $\begin{array}{c}\text { Questionário } \\
\text { de qualidade } \\
\text { de vida SF-36. }\end{array}$ & $\begin{array}{c}\text { Determinar a } \\
\text { prevalência e avaliar a } \\
\text { qualidade de vida em } \\
\text { mulheres casadas } \\
\text { incontinentes. }\end{array}$ & $\begin{array}{l}\text { A incontinência urinária é um } \\
\text { problema comum que afeta de } \\
\text { forma negativa a qualidade de } \\
\text { vida. E os principais fatores de } \\
\text { risco foram a infecção urinária } \\
\text { e idade avançada. }\end{array}$ \\
\hline $\begin{array}{c}\text { Análise } \\
\text { eletromiográfic } \\
\text { a e da qualidade } \\
\text { de vida na } \\
\text { incontinência } \\
\text { urinária }\end{array}$ & $\begin{array}{l}\text { SILVA, et al., } \\
2017 .\end{array}$ & $\begin{array}{c}14 \text { mulheres. } \\
\text { De } 21 \text { a } 29 \\
\text { anos. }\end{array}$ & $\begin{array}{c}\text { King's Health } \\
\text { Questionnaire } \\
\text { - KHQ. }\end{array}$ & $\begin{array}{l}\text { Avaliar a atividade } \\
\text { eletromiográfica dos } \\
\text { músculos do assoalho } \\
\text { pélvico e do reto } \\
\text { abdominal em mulheres } \\
\text { incontinentes. }\end{array}$ & $\begin{array}{c}\text { O estudo apontou pouca } \\
\text { interferência na qualidade de } \\
\text { vida, obtendo-se escores baixos } \\
\text { no questionário KHQ. Na } \\
\text { eletromiografia, percebeu-se } \\
\text { uma maior efetividade nas } \\
\text { amplitudes de contração de } \\
\text { fibras fásicas em ortostatismo e } \\
\text { de fibras tônicas em decúbito } \\
\text { dorsal. }\end{array}$ \\
\hline $\begin{array}{l}\text { Impacto dos } \\
\text { tipos de } \\
\text { incontinência } \\
\text { urinária na } \\
\text { qualidade de } \\
\text { vida de } \\
\text { mulheres. }\end{array}$ & $\begin{array}{l}\text { SABOIA, et al., } \\
2017 .\end{array}$ & $\begin{array}{c}685 \text { mulheres. } \\
\text { De } 22 \text { a } 89 \\
\text { anos. }\end{array}$ & $\begin{array}{c}\text { Medical } \\
\text { Outcomes } \\
\text { Study 36-item } \\
\text { Short-Form } \\
\text { Health Survey } \\
\text { (SF-36), } \\
\text { International } \\
\text { Consultation } \\
\text { Incontinence } \\
\text { Questionnaire } \\
\text { Short-Form } \\
\text { (ICIQ-SF), } \\
\text { King's Health } \\
\text { Questionnaire } \\
\text { (KHQ) e } \\
\text { Pelvic Organ } \\
\text { Prolapse } \\
\text { Incontinence } \\
\text { Sexual } \\
\text { Questionnaire } \\
\text { (PISQ-12). }\end{array}$ & $\begin{array}{l}\text { Comparar o impacto } \\
\text { dos diferentes tipos de } \\
\text { IU na QV geral e } \\
\text { específica destas } \\
\text { mulheres utilizando os } \\
\text { principais questionários } \\
\text { validados para o } \\
\text { português do Brasil. }\end{array}$ & $\begin{array}{l}\text { A pesquisa identificou que a } \\
\text { Incontinência urinária mista é a } \\
\text { que mais interfere } \\
\text { negativamente na vida das } \\
\text { mulheres que participaram do } \\
\text { estudo. }\end{array}$ \\
\hline $\begin{array}{l}\text { Avaliação da } \\
\text { qualidade de } \\
\text { vida em } \\
\text { mulheres com } \\
\text { sintomas de } \\
\text { incontinência } \\
\text { urinária de } \\
\text { esforço } \\
\text { praticantes de } \\
\text { atividade física. }\end{array}$ & $\begin{array}{l}\text { NAVES, et } \\
\text { al.,2016. }\end{array}$ & $\begin{array}{l}42 \text { mulheres } \\
\text { onde foram } \\
\text { selecionadas } \\
\text { apenas } 12 \\
\text { mulheres. De } \\
33 \text { a } 41 \text { anos. }\end{array}$ & $\begin{array}{c}\text { King's Health } \\
\text { Questionnaire } \\
\text { (KHQ). }\end{array}$ & $\begin{array}{l}\text { Identificar os sintomas } \\
\text { da incontinência } \\
\text { urinária de esforço em } \\
\text { mulheres praticantes de } \\
\text { atividade física, e a } \\
\text { qualidade de vida das } \\
\text { mesmas. }\end{array}$ & $\begin{array}{l}\text { O estudo sugere que as } \\
\text { atividades de impacto são fator } \\
\text { de risco importante para o } \\
\text { desenvolvimento da IUE, o que } \\
\text { pode influenciar de forma } \\
\text { negativa na qualidade de vida } \\
\text { destas mulheres. }\end{array}$ \\
\hline $\begin{array}{l}\text { Caracterização } \\
\text { e fatores de } \\
\text { risco de } \\
\text { Incontinência } \\
\text { urinária em } \\
\text { mulheres } \\
\text { atendidas em } \\
\text { uma clínica } \\
\text { ginecológica. }\end{array}$ & $\begin{array}{l}\text { MOURÃO, et al., } \\
2016 .\end{array}$ & $\begin{array}{c}48 \text { mulheres. } \\
\text { De } 30 \text { a } 49 \\
\text { anos }\end{array}$ & $\begin{array}{c}\text { Formulário } \\
\text { semiestruturad } \\
\text { o. }\end{array}$ & $\begin{array}{l}\text { Caracterizar o perfil } \\
\text { sociodemográfico, } \\
\text { epidemiológico e } \\
\text { clínico de mulheres com } \\
\text { IU atendidas em uma } \\
\text { clínica ginecológica de } \\
\text { um hospital } \\
\text { universitário. }\end{array}$ & $\begin{array}{l}\text { Os principais fatores de risco } \\
\text { para IU apresentados foram: } \\
\text { idade, consumo de café, } \\
\text { histórico de cirurgias } \\
\text { ginecológicas e de infecções } \\
\text { recorrentes do trato urinário } \\
\text { inferior, obesidade, cafeína, } \\
\text { prática de atividade física. }\end{array}$ \\
\hline $\begin{array}{l}\text { Incontinência } \\
\text { Urinária em } \\
\text { mulheres } \\
\text { jovens } \\
\text { praticantes de } \\
\text { exercício físico. }\end{array}$ & $\begin{array}{l}\text { PATRIZZI, et } \\
\text { al.,2014. }\end{array}$ & $\begin{array}{l}\text { Aplicado em } \\
108 \text { mulheres. } \\
\text { De } 18 \text { a } 30 \\
\text { anos. }\end{array}$ & $\begin{array}{l}\text { Questionário } \\
\text { estruturado. }\end{array}$ & $\begin{array}{l}\text { Comparar a prevalência } \\
\text { do autorrelato de } \\
\text { incontinência urinária } \\
\text { em mulheres jovens de } \\
\text { acordo com as } \\
\text { modalidades de } \\
\text { exercício físico. }\end{array}$ & $\begin{array}{c}\text { Foi visto que a incontinência } \\
\text { urinária está presente em } \\
\text { grande porcentagem nas } \\
\text { diferentes modalidades } \\
\text { esportivas. }\end{array}$ \\
\hline
\end{tabular}

Fonte: Souza, Souza, Dantas e Alencar (2021). 
De acordo com, Fernandes et al., (2020), o aumento da idade é apontado como um fator de risco significativo, devido a redução dos níveis de estrogênio que leva a atrofia da musculatura uretral, contudo a incontinência urinária não é específica de mulheres idosas, visto que o número de mulheres jovens com essa disfunção tem se tornado relevante.

Corroborando com esta ideia Mourão, et al., (2016) afirmou em seu estudo que a incontinência urinária é uma patologia que afeta as mulheres em diversas fases do ciclo vital, sendo que o pico de mulheres jovens afetadas pela doença pode ocorrer na faixa etária de 30 à 49 anos, onde os principais fatores de risco são multiparidade, obesidade, consumo excessivo de cafeína , diabetes, infecção urinária e a prática de atividades físicas de impacto, sendo a baixa escolaridade e o fator financeiro fatores que levam as pacientes a não buscarem serviços de saúde, pois notou-se que 45,8\% das participantes da pesquisa tem renda individual de até 1 salário mínimo, ponderando que coabitam com 3 ou mais pessoas, sendo inviável para o sustento familiar e manutenção da saúde.

Em contrapartida aos estudos discutidos anteriormente que frisavam a questão socioeconômica, Patrizzi, et al., (2014) abordou em seu estudo que mulheres jovens praticantes de exercícios de alto impacto, com ênfase na modalidade musculação podem atingir o mecanismo de incontinência devido ao aumento da pressão intra-abdominal, sobrecarregando a musculatura do assoalho pélvico e assim desencadeando fadiga desta musculatura. Seguindo este raciocínio, Naves et al., (2016) agrega que é importante incluir exercícios para fortalecimento da musculatura do assoalho pélvico (MAP) durante as atividades físicas, levando em consideração a importância da prática de atividade física para manutenção da saúde, fazendo com que mulheres tenham adequado condicionamento do MAP, bem como melhor qualidade de vida.

Em relação a esse aspecto Silva, et al., (2017) apontou que maior parte das mulheres não compreendem a importância do fortalecimento da musculatura do assoalho pélvico. Interferindo e prejudicando o seu tratamento, e qualidade de vida, foi verificado por Dantas, et al., (2020), que nenhuma das 300 mulheres participantes de seu estudo não procurou quaisquer atendimentos de saúde para a disfunção de incontinência urinaria, visto assim que a falta de conhecimento sobre a patologia, prevenção e tratamento adequado tendem a ter um diagnóstico incompleto devido escassez da procura ao atendimento médico.

Embora a Incontinência urinária não seja um questão de saúde fatal, ela é um problema que afeta de forma negativa a qualidade de vida das mulheres, onde limita as atividades de vida diária das mesmas, fator que, segundo Dedicação, et al., (2018), tem influência sobre as relações sociais e a percepção própria de saúde, o mesmo estudo aponta que os maiores problemas estão relacionados ao bem estar social e mental, afetando relações sexuais, causando isolamento social, baixa autoestima e depressão.

Ainda abordando sobre qualidade de vida, Ozdemir, et al., (2018), afirma que a mesma foi vista como pior em mulheres com histórico de incontinência urinária do que aquelas sem histórico dessa patologia, onde as mais prejudicadas são aquelas que possuem incontinência urinária mista (IUM). Seguindo o mesmo raciocínio que segundo do estudo anterior Saboia, et al., (2017), aponta que a associação dos sintomas de urgência e esforço podem levar a maiores limitações na vida diária quando comparada aos sintomas isolados, o que se justifica o fato de que mulheres com IUM apresentam os piores escores na avaliação da qualidade de vida, uma vez que sintomas se intensificam.

\section{Conclusão}

A qualidade de vida tem recebido uma importância maior nos últimos anos, no que diz respeito a saúde, não somente por apresentar uma abordagem complexa sobre a percepção do indivíduo, mas também por permitir avaliar fatores físicos, psicológicos e sociais.

Em virtude disso, apesar de serem escassos o número de estudos encontrados, através dos estudos utilizados, podemos concluir que a incontinência urinária afeta de forma negativa a qualidade de vida das mulheres jovens, principalmente no que diz respeito ao âmbito psicológico, incapacitando-as de realizar de forma adequada suas atividades de vida diária. 
Dessa forma, sugere-se que sejam feitas novas pesquisas em relação ao tema, pois são de grande importância para o desenvolvimento científico, principalmente com o público mais jovem, levando em consideração que as mulheres jovens também são afetadas pela incontinência urinária, haja vista, que a maioria dos estudos que estão na literatura abrange o público mais idoso, impossibilitando assim a obtenção de um melhor estudo literário, para assim agregar mais conhecimento com relação ao tema a toda comunidade acadêmica e profissional que tenha interesse pela temática.

\section{Agradecimentos}

Primeiro agradecemos a Deus por conseguir concluir mais uma etapa em nossas vidas. Segundo, agradecemos nossas famílias, que sempre nos apoiaram e nos incentivaram durante a árdua jornada acadêmica, também lembrar daqueles familiares que hoje não estão entre nós, mas que nos deram forças para realizar o sonho da graduação, que antes parecia distante da nossa realidade. Agradecemos a nossa orientadora por, sempre, ser solícita e presente, por nos guiar e apoiar nossas decisões. Tê-la como orientadora foi muito além da relação professora e acadêmicas, pois tornou-se amiga e referência profissional. A parceria selada entre nossos amigos de graduação durante todos esses anos, com certeza, ficará na memória: os risos, discussões, lágrimas e as confraternizações. Por fim, é gratificante perceber que apesar de tudo que passamos a graduação só nos fez mais fortes e perseverantes em nosso propósito: Nos tornar fisioterapeutas conscientes da importância da atuação da nossa profissão para sociedade.

\section{Referências}

Abrams, P., Cardozo, L., Khoury, S. \& Wein, A. (2013). Incontinence.5th Edition ICUD-EAU eds. Layout: SMART-DOT marketing \& web consultants, www.smart-dot.com. 15-107.

Berghmans, B., Morkved, S. \& Van Kampen, M. (2015). Evidence-based Physical Therapy for the pelvic floor: Bridging science and clinical practice. (2nd ed.), 224-226.

Dantas, M. A., Dias, C. \& Nascimento, E. G. C. (2020). Frequência da incontinência urinária em mulheres na idade produtiva. Revista Enfermagem Atenção Saúde. 16-27.

Dedicação, A. C., Haddad, M., Saldanha M. E. S. \& Driusso, P. (2018). Comparação da qualidade de vida nos diferentes tipos de incontinência urinária feminina. Revista Brasileira de Fisioterapia. 13(2): 1-7 https://doi.org/10.1590/S1413-35552009005000014.

Delarmelindo, R. D. C. A., Parada, C. M. G. D. L., Rodrigues, R. A. P., \& Bocchi, S. C. M. (2012). Estratégias de enfrentamento da incontinência urinária por mulheres. Revista da Escola de Enfermagem da USP, 47(2), 296-303.

Fernandes, C. \& Ognibeni, L. C. R. (2020). Prevalência e Fatores de risco associados a incontinência urinária em acadêmicas do curso de fisioterapia. Revista Uningá. 58, 32-33.

Fernandes, S., Coutinho, E., Duarte, J., Nelasp., Chaves, C. \& Amaral, O. (2015). Qualidade de vida em mulheres com incontinência urinária. Revista de Enfermagem. 4, 93-99.

Leroy, L., Lopes, M. \& Shimo, A. (2012). A incontinência urinária em mulheres e os aspectos raciais: uma revisão de literatura. Revista Literária. Florianópolis.21(3) 692-700.

Liao, Y. M., Yang, C. Y., Kao, C. C., Dougherty, M. C., Lai, Y. H. \& Chang, Y. (2009). Prevalence and impact on quality of life of lower urinary tract symptoms among a sample of employed women in Taipei: aquestionnaire survey. Int J Nurs Stud. 46(5):633-44.

Marques, F. K. \& Freitas, C. P. (2017). A cinesioterapia como tratamento da incontinência urinária na unidade básica de saúde. Revista Fisioterapia em Movimento. 18(4).

Mourão, L. F. et al. (2017). Caracterização e fatores de risco de incontinência urinária em mulheres atendidas em uma clínica ginecológica. Revista Estima. Teresina-PI. 15(2), 82-91.

Naves, P. P., Letieri, R. V., Simon, I. S. L., Leite, S. N. \& Letieri, M. (2016). Avaliação da qualidade de vida em mulheres com sintomas de incontinência urinária de esforço praticantes de atividade física. Revista Inspirar, Movimento \& Saúde. Minas Gerais. Edição $37-$ vol. 8 -n.1.

Oliveira, J., Gracia, R. (2011). Cinesioterapia no tratamento de incontinência urinária em mulheres idosas. Revista Brasileira de Geriatria e Gerontologia. 14, 343-351.

Ozdemir, K., Sahin, S., Ozerdogan, N. \& Unsal, A. (2018). Evaluation of urinary incontinence and quality of life in married women aged between 20 and 49 years. Turkish jornal of Medical Sciencs. 48, 100-109. 
Research, Society and Development, v. 10, n. 13, e23101321033, 2021

(CC BY 4.0) | ISSN 2525-3409 | DOI: http://dx.doi.org/10.33448/rsd-v10i13.21033

Patrizzi, L. J., Viana, D. A., Silva, L. M. A. \& Pegorari, M. S. (2014). Incontinência urinária em mulheres jovens praticantes de exercício físico. Revista Bras. Ci. e Mov. 22(3): 105-110.

Pedro, A., Ribeiro, J., Soler, Z. \& Bugdan, A. (2011). Qualidade de vida de mulheres com incontinência urinária. Rev. Eletrônica Saúde Mental Álcool Drog. (Ed. port.) 7, 2.

Pereira, A. S., Shitsuka, D. M., Parreira, F. J. \& Shitsuka, R. (2018). Ciência e conhecimento científico. Metodologia da pesquisa científica.

Rett, M. T., Simões, J. A., Herrmann, V., Gurgel, M. S. C. \& Morais, S. S. (2007). Qualidade de vida em mulheres após tratamento da incontinência urinária de esforço com Fisioterapia. Revista Brasileira Ginecologia Obstetrícia. 29(3):134-40.

Saboia, D.M., Firmiano, M. L.V., Bezerra, K. C., Vasconcelos, J. A., Oriá, M. O. B. \& Vasconcelos, C. T. M. (2017). Impact of urinary incontinence types on women's quality of life. Revista Escola de Enfermagem USP. 2017;51:e03266. http://dx.doi.org/10.1590/S1980-220X2016032603266.

Salama, S., Boitrelle, F., Gauquelin, A., Malagrida, L., Thiounn, N. \& Desvaux P. (2015). Natureza e origem de "esguichar" na sexualidade feminina. J Sex Med. 12, 661-6.

Silva, S. C. S., et al. (2017). Análise eletromiográfica e da qualidade de vida na incontinência urinária. Revista Fisioterapia Brasil. Parnaíba. 18(5): 608-615. 